\title{
Only a small proportion of patients with first episode psychosis come via prodromal services: a retrospective survey of a large UK mental health programme
}

Olesya Ajnakina 1* (D), Craig Morgan², Charlotte Gayer-Anderson², Sherifat Oduola, François Bourque², Sally Bramley ${ }^{4}$, Jessica Williamson ${ }^{5}$, James H. MacCabe ${ }^{1,6}$, Paola Dazzan ${ }^{1,6}$, Robin M. Murray ${ }^{1}$ and Anthony S. David ${ }^{1}$

\begin{abstract}
Background: Little is known about patients with a first episode of psychosis (FEP) who had first presented to prodromal services with an "at risk mental state" (ARMS) before making the transition to psychosis. We set out to identify the proportion of patients with a FEP who had first presented to prodromal services in the ARMS state, and to compare these FEP patients with FEP patients who did not have prior contact with prodromal services.

Methods: In this study information on 338 patients aged $\leq 37$ years who presented to mental health services between 2010 and 2012 with a FEP was examined. The data on pathways to care, clinical and socio-demographic characteristics were extracted from the Biomedical Research Council Case Register for the South London and Maudsley NHS Trust.

Results: Over 2 years, 14 (4.1\% of $n=338$ ) young adults presented with FEP and had been seen previously by the prodromal services. These ARMS patients were more likely to enter their pathway to psychiatric care via referral from General Practice, be born in the UK and to have had an insidious mode of illness onset than FEP patients without prior contact with the prodromal services.
\end{abstract}

Conclusions: In the current pathways to care configuration, prodromal services are likely to prevent only a few at-risk individuals from transitioning to psychosis even if effective preventative treatments become available.

Keywords: First episode psychosis, At risk mental state, Pathways to care, Retrospective, Transition to psychosis, Prodromal services

\section{Background}

Psychotic disorders and schizophrenia in particular historically are progressive deteriorating conditions. A long duration of untreated psychosis (DUP) is believed to be an important contributing factors to the severity of the illness and subsequent poorer outcomes [1]. This has added to the focus on specialist early intervention (EI) services for first episode psychosis (FEP) $[2,3]$ the aim

\footnotetext{
* Correspondence: olesya.ajnakina@kcl.ac.uk

${ }^{1}$ Department of Psychosis Studies, Institute of Psychiatry, Psychology \& Neuroscience, King's College London, 16 De Crespigny Park, London SE5 $8 \mathrm{AF}, \mathrm{UK}$

Full list of author information is available at the end of the article
}

of which is to reduce treatment delay, and thus to increase chances for recovery and to improve overall prognosis of psychosis [1]. Even though some studies have suggested that the benefits of EI services may be negligible [4], the British EI services are designed to intervene during the first three years after the illness onset [5] - a period that is perceived to be crucial in shaping the prognosis of the illness [6] - by means of administering low doses of antipsychotics, family involvement, education and support [5]. There is also evidence showing that EI indeed reduces DUP once patients entered the programme [7]. In the UK pathways to care for FEP are diverse. The most widely 
accepted route of access to mental health services is through general practitioners (GPs), who then refer the patients to specialist psychiatric services [8]. However, more often than not, other non-healthcare agencies such as police and the criminal justice system serve as the first point of contact for individuals in crisis with their first onset of psychosis [8].

The FEP is often (but not always) preceded by a phase termed the At Risk Mental State (ARMS) [9], which is characterised by either 'attenuated' psychotic symptoms, or full blown psychotic symptoms that are brief and selflimiting. The ARMS period may also manifest as a significant decrease in functioning in the context of a genetic risk for schizophrenia or subtle subjective disturbances of cognitive processes, changes in thinking, perception, mood, affect and behaviour [10, 11]. Up to $30-45 \%$ of those with the ARMS are claimed to develop a psychotic disorder in the following 24 months [12]; though with some reporting transition rates $11-17 \%$ within a 2-year period [13, 14]. Identification of the ARMS individuals therefore provides a unique opportunity to attempt to prevent such individuals transitioning to clinical psychosis $[1,9,15]$. This recognition has provoked the development of prodromal services that provide comprehensive care to young individuals meeting criteria for the ARMS [9, 11, 16, 17]. Having established close links with primary care providers and non-health related community services, such as schools, counsellors, and emergency and criminal justice agencies, prodromal services provide an accessible and acceptable service for help-seeking young people who are at risk of psychosis $[9,18]$.

Surprisingly, to our knowledge there has been no published report from anywhere in the world on the proportion of individuals who present to psychiatric services with FEP and who have previously accessed mental health care via prodromal services. Compared to the standard EI services, access to the prodromal services requires patients to demonstrate active help-seeking $[9,18]$, a requirement that may render individuals who attend such services unrepresentative of the overall at risk population. Moreover, knowledge of pathways to care for those at high risk of psychosis remains incomplete [19] as does how such pathways differ from those used by the remainder of patients with a FEP [20]. A better understanding of how different groups interact with healthcare systems may enable such systems to more effectively target interventions aimed at preventing transition to clinical psychosis in those with an ARMS [20].

The aims of the present study are two-fold. First, we set out to identify the proportion of patients with FEP who had first presented to the local prodromal services in the ARMS state before making the transition to FEP. Second, we sought to compare clinical and socio-demographic characteristics and differences in pathways to care between such FEP patients and those FEP patients presenting directly to standard first episode services, all within a tightly defined catchment area served by the South London and Maudsley NHS Foundation Trust (SLaM). We hypothesised that the interval between the first onset of symptoms and initiation of treatment would be shorter in those attending the prodromal services compared with those patients referred to conventional services. Furthermore, although a number of earlier studies have shown that a number of patients who are referred to the prodromal services are already experiencing a first episode of psychosis at the time of the contact $[9,21]$, it is not known whether this subgroup of patients differs from FEP patients who present to generic services, perhaps because of factors related to help-seeking. We additionally investigated this question by comparing the those individuals who were referred to prodromal services with a suspected ARMS but in fact were already experiencing a full clinical first psychotic episode with those FEP patients who did not have prior contact with the prodromal services before developing a FEP in terms of sociodemographic characteristics, duration of untreated psychosis, clinical presentation and pathways to care.

\section{Methods}

Sample

Patients in this study were identified as part of the European Union Gene-Environment Interactions (EU-GEI) study and presented to mental health services in the South London and Maudsley NHS Foundation (SLaM) Trust between 1 May 2010 to 30 April 2012 with a FEP (International Classification of Diseases [ICD-10] codes F20-F29 and F30-F33) (World Health Organisation [WHO], 1992) validated by administration of the Schedules for Clinical Assessment in Neuropsychiatry [22]. The age limit in this study was 37 years old and was chosen to be consistent with the age limit for patients attending the ARMS clinic (35 years) plus 2 years to allow them to present with a FEP. The patients were included in the study if they were current residents of Lambeth (population 303,086) or Southwark (population 288,238) [23] boroughs served by the Trust. Exclusion criteria were: 1) evidence of psychotic symptoms precipitated by an organic cause; 2 ) transient psychotic symptoms resulting from acute intoxication as defined by ICD-10; and 3) head injury causing clinically significant loss of consciousness. No specific screening criteria were used.

The FEP patients were further subdivided into 3 groups: 1) FEP patients who had first presented to the prodromal services with the ARMS and who, by definition, subsequently transitioned to FEP (i.e., $P R O D$ group); 2) FEP patients without prior contact with the prodromal services before their first contact with the mental health services for FEP (i.e., FEP-C (control) 
group); and 3) patients who were found to be already experiencing their first psychotic episode at the time of first contact with the prodromal services (i.e., FEP-P (psychosis) group).

\section{Data source}

The patients for this study were identified from electronic records obtained from the SLaM Biomedical Research Centre (BRC) Case Register Interactive Search tool (CRIS). The SLaM, whose case records provide the source data for the SLaM BRC Case Register, is the largest provider of secondary mental healthcare in Europe, covering a socially diverse region of 1.2 million residents in South East London [24]. Within the UK National Health Service context, effectively all secondary mental health services are managed and provided by single, geographicallydefined mental health trusts (such as SLaM).

CRIS was developed in 2008 and consists of a series of data-processing algorithms which effectively anonymise [25], structure and extract all electronic health records, including correspondence, discharge letters and events, reported by treating clinicians throughout patients' journeys through the Trust services [24-26]. Using the CRIS system, we identified all patients who came in contact with the SLaM for a FEP over 2 year period. Where there was ambiguity about the FEP status of a patient, a consensus decision was made by members of the research team; this always included those with longstanding expertise in first episode psychosis (C.M.). The CRIS was further utilised to extract information on socio-demographic characteristics, clinical presentation and pathways to care for all identified patients. Additionally, we used CRIS to identify those patients from our cohort of FEP cases who were referred to the Outreach and Support in South London Service (OASIS) $[9,17]$ services and who, having met criteria for the ARMS (using the Comprehensive Assessment of the At Risk Mental State (CAARMS) [27]), were accepted for treatment prior to making the transition to FEP.

A comprehensive description of these EI services and how they operate is provided elsewhere $[9,17]$. Briefly, OASIS is a specialised community mental health service for people aged 14-35 years old with the ARMS for psychosis. It is one of the largest and most well established prodromal services in the world and is well integrated with the first-episode services in all main South London boroughs. OASIS accepts referrals via telephone, mail and fax, which can be made by individuals themselves, their friends, relatives, mental or non-mental health professionals as well as nonhealth agencies including educational establishments, community services and churches. OASIS team responds promptly to all referrals and conducts the first assessment within the first week of the referral being made and provides a 2-year follow-up to those with ARMS [18].

\section{Assessments \\ Socio-demographic characteristics}

The Medical Research Council (MRC) Socio-demographic Schedule (modified version) was utilised to extract data on socio-demographic characteristics and cannabis use [28]. Ethnicity was self-ascribed and recorded on the clinical notes by the treating clinicians and was further classified using the 18 categories employed by the 2011 UK Census (http://www.ons.gov.uk/census/2011census). Due to relatively small numbers of patients in some of our groups, we combined the ethnic categories into three broad ethnic groups: white (all white groups), black (all black groups), and other (encompassing Asian, mixed and other ethnicities).

\section{Clinical presentation}

Duration of untreated psychosis (DUP) was defined as the time between the date of an appearance of first symptoms of psychosis and date of start of first treatment with antipsychotic medications [29]. Age at first contact was defined as the age at which a patient was in contact with mental health services for the first time [30]. Similar to previous studies [31, 32], mode of onset of psychotic symptoms was operationalised using definitions developed for the World Health Organisation International Pilot Study of Schizophrenia, and was categorised into three groups: 1) acute (psychotic symptoms appeared within hours, 1 week or 1 month of first noticeable behavioural change); 2) gradual (psychotic symptoms appeared within period of 1 to 6 months of first noticeable behavioural change); and 3) insidious (psychotic symptoms appeared incrementally over a period of 6 months or greater since first noticeable behavioural change).

\section{Pathways to care}

The four most commonly used pathways were examined: 1) general practitioner (GP); 2) emergency medical services (primarily hospital accident and emergency departments, and walk-in centres); 3) criminal justice agencies (police, prison or probation services and courts); and 4) health workers (social support workers, nurses or other mental health workers).

\section{Statistical analysis}

The distributions of socio-demographic characteristics, clinical presentation and pathways to care were explored with frequencies, percentages, mean and standard deviation, median and inter-quartile range (IQR). The comparisons between the groups were made using $x^{2}$ test or Fisher's exact test when expected cell counts were 
less than 5 [33] for categorical data and t-tests for continuous data. DUP was heavily skewed and was consequently log-transformed to allow parametric analyses. DUP for each group of patients is presented in the original scale, while the analyses were conducted using the logarithmic-transformed values. To test the differences in clinical presentation and pathways to care between the groups of patients on their first contact with the services independent of confounding factors we employed exact logistic regression (ELR), which is an ideal and methodologically logical alternative approach to the maximum unconditional likelihood method when sample sizes are small or the data are sparse [34]. As it has been shown that in order to maintain the validity of the models the ratio of the number of patients suffering endpoints to the number of potential predictors should be at least 10:1 [35, 36], we adjusted our ELR models for one confounding variable; that is age at first contact with mental health services. All analyses were conducted in STATA release 14 (STATACorp, USA).

\section{Results}

\section{Sample characteristics}

The information on the groups is illustrated in the Fig. 1. Between 2010 and 2012, there were 338 referrals aged $\leq 37$ years old for first episode psychosis within catchment boroughs served by the SLaM Trust. Of these, 283 (83.7\%) were referred to conventional mental health services for FEP without prior contact with the prodromal services (i.e., FEP-C group). Overall, 55 (16.3\% of 338) FEP patients had been in contact with the prodromal services. Of these, 14 ( $25.5 \%$ of 55 and $4.1 \%$ of 338 FEP cases) met criteria for the ARMS and subsequently transitioned to FEP (i.e., PROD group); the remaining 41 (74.5\% of 55 and $12.1 \%$ of 338 ) already met criteria for a clinical psychotic disorder at the time of first contact with the prodromal services (i.e., FEP-P group).

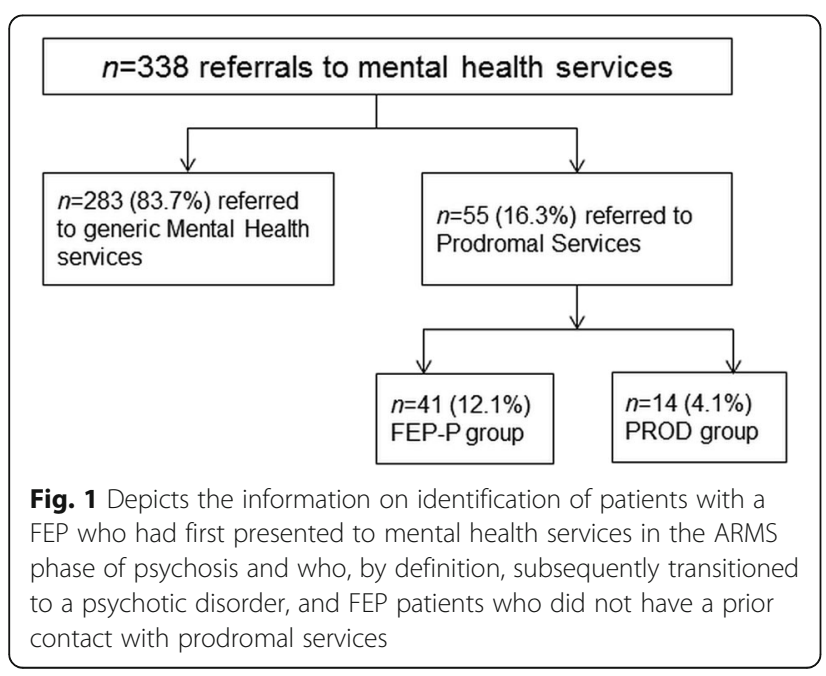

Socio-demographic characteristics: FEP-C vs PROD groups Comparisons in socio-demographic characteristics between the FEP-C and PROD groups at the time of first contact with mental health services are presented in Table 1. At the time of first contact with mental health services, the FEP-C group was older (mean $=27.9$ years, S.D. $=5.5)$ than the PROD group (mean $=24.2$ years, S.D. $=6.0)(t(295)=2.46, P=0.01)$. A higher proportion of the PROD group ( $n=10 / 14,83.3 \%$ ) was born in the UK compared with the FEP-C group ( $n=124 / 234,47 \%)$ $\left(x^{2}=6.14, P=0.01\right)$.

\section{Clinical presentation and pathways to care: FEP-C vs PROD groups}

Comparisons in clinical presentation and pathways to care between the FEP-C and PROD groups at the time of first contact with mental health services are presented in Table 2. DUP was highly skewed; the median length of DUP was substantially longer in the FEP-C group ( median $=86$ day, IQR $=13-368)$ compared with the prodromal group (median $=19$ day, IQR $=6-40$ ), albeit the difference was not statistically significant at the conventional level of $P<0.05(t(219)=1.35, P=0.18)$. Further, $73 \%(n=8 / 11)$ of the PROD group and $36 \%$ ( $n=100 / 277$ ) of the FEP-C group had an insidious mode onset. Indeed, the PROD group was more likely to have an insidious mode of psychosis symptoms onset compared to those patients with FEP who did not have prior contact with prodromal services $(\mathrm{OR}=5.17$, 95\% $\mathrm{CI}=1.18-31.51)$. Moreover, the pathways to care were varied among the groups: $45 \%(n=124 / 274)$ of the FEP$\mathrm{C}$ group made first contact with mental health services via emergency medical services and $18 \%(n=49 / 274)$ of this group were referred by the criminal justice system. By contrast, $77 \%(n=10 / 13)$ of the PROD group entered their pathway to care via referral from GPs or other health professional. FEP patients who had first presented to the prodromal services with the ARMS and who subsequently transitioned to FEP were less likely to make their first contact with mental health services via emergency services compared to those FEP patients who did not have prior contact with prodromal services $(\mathrm{OR}=0.19,95 \% \mathrm{CI}=0.02-0.91)$.

\section{Socio-demographic characteristics, DUP and pathways to care: FEP-C vs FEP-P groups}

Of all $55(16.3 \%$ of $n=338)$ FEP patients who had prior contact with the prodromal services, $75 \%(n=41 / 55)$ were already experiencing a full psychotic episode at the time of first contact with prodromal services (i.e., FEPP). This group of patients was younger ( mean $=24.7$ years, S.D. = 4.4) than those FEP patients who did not come in contact with the prodromal services prior to developing a psychotic disorder $($ mean $=27.9$ years, S.D. $=5.5)$ 
Table 1 Comparisons in socio-demographic variables between FEP-C (control) and PROD (prodrome) groups

\begin{tabular}{|c|c|c|c|c|c|}
\hline \multirow[t]{2}{*}{ Socio-demographic characteristics } & \multirow{2}{*}{$\begin{array}{l}\text { FEP-C }(n=283 ; 83.7 \%) \\
\text { Mean (S.D.) } / n(\%)\end{array}$} & \multirow{2}{*}{$\begin{array}{l}\text { PROD } \\
(n=14 ; 4.1 \%) \\
\text { Mean (S.D.)/n(\%) }\end{array}$} & \multicolumn{3}{|l|}{ Statistics } \\
\hline & & & Test statistics & df & $P$-value \\
\hline Age, y & $27.9(5.5)$ & $24.2(6.0)$ & $t=2.46$ & 295 & 0.01 \\
\hline Range & $18-37$ & $15-34$ & & & \\
\hline Gender & & & $\mathrm{chi}^{2}=0.36$ & 1 & $0.60^{\mathrm{a}}$ \\
\hline Female & $124(43.8)$ & $5(35.7)$ & & & \\
\hline Male & $159(56.2)$ & $9(64.3)$ & & & \\
\hline Ethnicity & & & $c h i^{2}=2.65$ & 2 & $0.29^{\mathrm{a}}$ \\
\hline White & $98(35.1)$ & $7(53.8)$ & & & \\
\hline Black & $126(45.2)$ & $3(23.1)$ & & & \\
\hline Other & $55(19.7)$ & $3(23.1)$ & & & \\
\hline Country of birth & & & $\mathrm{chi}^{2}=6.14$ & 1 & $0.02^{\mathrm{b}}$ \\
\hline UK & $124(46.8)$ & $10(83.3)$ & & & \\
\hline Not in the UK & $141(53.2)$ & $2(16.7)$ & & & \\
\hline Education & & & $\mathrm{chi}^{2}=1.30$ & 1 & $0.21^{b}$ \\
\hline School & $111(55.2)$ & $8(72.7)$ & & & \\
\hline A-Level, or above & $90(44.8)$ & $3(27.3)$ & & & \\
\hline Employment status & & & $\mathrm{chi}^{2}=1.24$ & 1 & $0.27^{\mathrm{a}}$ \\
\hline Unemployed & $172(64.7)$ & $7(50.0)$ & & & \\
\hline Employed & $94(35.3)$ & $7(50.0)$ & & & \\
\hline Marital status & & & $\operatorname{chi}^{2}=2.98$ & 1 & $0.10^{\mathrm{a}}$ \\
\hline Not in stable relationship & $207(75.3)$ & $7(53.9)$ & & & \\
\hline Married/stable relationship & $68(24.7)$ & $6(46.1)$ & & & \\
\hline Living arrangements & & & $\operatorname{chi}^{2}=4.08$ & 2 & $0.15^{b}$ \\
\hline Alone & $74(27.0)$ & $1(7.1)$ & & & \\
\hline Partner/family & $125(45.6)$ & $10(71.4)$ & & & \\
\hline No stable accommodation & $75(27.4)$ & $3(21.4)$ & & & \\
\hline Cannabis use & & & $\mathrm{chi}^{2}=1.86$ & 1 & $0.26^{b}$ \\
\hline No & $118(50.2)$ & $4(30.8)$ & & & \\
\hline Yes & $117(49.8)$ & $9(69.2)$ & & & \\
\hline
\end{tabular}

ARMS At Risk Mental State, IQR inter-quantile range, S.D. standard deviation, $d f$ degree of freedom, $y$ years, FEP-C (control) group FEP patients who present to mental health services for FEP without prior contact with the prodromal services, $P R O D$ (prodrome) group FEP patients who had first presented to the prodromal services with the ARMS and who, by definition, subsequently transitioned to FEP ${ }^{a}$ chi-square test

${ }^{\mathrm{b}}$ Fisher's exact test

$(t(322)=3.56, P<0.001)$. On the first contact with mental health services, 63\% $(n=26 / 41)$ of the FEP-P group lived with members of their family or partners compared to $46 \%$ ( $n=125 / 274)$ of the FEP patients without a prior contact with the prodromal services (i.e., FEP-C group) $\left(x^{2}=6.77, P=0.03\right)$ (Additional file 1). Further, the pathways to care differed among these two groups: $46 \%$ ( $n=18 / 39)$ of the FEP-P group were referred to mental health services by their local GPs, while 45\% ( $n=124$ / 274 ) of the FEP-C group were referred by the emergency medical services; $17.9 \%(n=49 / 274)$ of the FEP-C group, compared to $7.7 \%(n=3 / 39)$ of the FEP-P group, came in contact with mental health services via the criminal justice agencies. The results of the exact logistic regression analysis highlighted that the FEP-P group was more likely to make the first contact with mental health services via GPs when compared to those patients who did not have prior contact with the prodromal services (Additional file 2).

\section{Discussion}

We found that $4.1 \%$ of patients presenting to mental health services with first episode psychosis (FEP) had previously presented to the prodromal services with the 
Table 2 Comparisons in clinical presentation characteristics and pathways to care between FEP-C (control) and PROD (prodrome) groups

\begin{tabular}{|c|c|c|c|c|}
\hline \multirow[t]{2}{*}{ Clinical presentation and pathways to care } & \multirow{2}{*}{$\begin{array}{l}\text { FEP-C }(n=283 ; 83.7 \%) \\
\text { Median }(\mathrm{IQR}) / \mathrm{n}(\%)\end{array}$} & $\operatorname{PROD}(n=14 ; 4.1 \%)$ & \multirow[t]{2}{*}{ Unadjusted OR (95\% Cl) } & \multirow[t]{2}{*}{ Adjusted $^{\mathrm{a}} \mathrm{OR}(95 \% \mathrm{Cl}$} \\
\hline & & Median (IQR)/n(\%) & & \\
\hline DUP, $d$ & $86(13-368)$ & $19(6-40)$ & $0.99(0.96-1.00)$ & $0.99(0.96-1.00)$ \\
\hline \multicolumn{5}{|l|}{ Source of referral } \\
\hline General Practitioner & $67(24.4)$ & $6(46.2)$ & $2.64(0.71-9.53)$ & $2.82(0.75-10.34)$ \\
\hline Emergency services & $124(45.3)$ & $2(15.4)$ & $0.22(0.02-1.04)$ & $0.19^{*}(0.02-0.91)$ \\
\hline Health \& social worker & $34(12.4)$ & $4(30.8)$ & $0.38(0.01-2.71)$ & $0.43(0.01-3.10)$ \\
\hline Criminal justice agency & $49(17.9)$ & $1(7.7)$ & $3.12(0.66-11.96)$ & $3.23(0.68-12.68)$ \\
\hline \multicolumn{5}{|l|}{ Mode of onset } \\
\hline Acute & $121(43.7)$ & $3(27.3)$ & $0.48(0.08-2.07)$ & $0.42(0.07-1.85)$ \\
\hline Gradual & $56(20.2)$ & - & $0.26(0.00-1.63)$ & $0.28(0.00-1.80)$ \\
\hline Insidious & $100(36.1)$ & $8(72.7)$ & $4.69^{*}(1.10-28.09)$ & $5.17^{*}(1.18-31.51)$ \\
\hline
\end{tabular}

DUP duration of untreated psychosis, GP general practitioner, IQR 25th and 75th Percentiles range, S.D. standard deviation, $d f$ degree of freedom, $d$ days, $C I$ confidence intervals, FEP-C (control) group FEP patients who present to mental health services for FEP without prior contact with the prodromal services, PROD (prodrome) group FEP patients who had first presented to the prodromal services with the ARMS and who, by definition, subsequently transitioned to FEP ${ }^{*} p<0.05,{ }^{* *} p<0.01,{ }^{* * *} p<0.001$

${ }^{a}$ Adjusted for age at the first contact with mental health services

ARMS and subsequently transitioned to a psychotic disorder. Although such services are well-known locally, it may be that recognising appropriate cases and directing them to the prodromal service is simply beyond the skills and training of most referrers [37]. The task of effectively detecting the true ARMS cases based on referrals and help-seeking behaviour rather than epidemiological surveys is clearly challenging especially given the lack of sensitive and specific biomarkers indicative of the prodromal phase of psychosis $[38,39]$. If these figures are replicated in other settings with similar prodromal services, this will suggest that the promise of early detection of those at high risk for transition to clinical psychosis with the aim of primary prevention, on a large scale, is still some way off. Even if effective, safe, acceptable and efficient interventions were readily available, our findings indicate that we are not yet in a position to apply them in a way which could make anything but a small impact on the incidence of psychosis.

\section{How could this very low figure be improved?}

We found that around $77 \%$ of all referrals to the prodromal services were made by health professionals such as local general practitioners (GPs) and other health workers. Clearly, this pathway leaves out young individuals developing psychosis who do not seek help [40,41] or are not registered with GPs. Similarly, migrants may be less likely to be registered with GPs and may have less trusting attitudes toward mental health professionals [42]. Indeed, in the present study we found that most prodromal patients who came in contact with the prodromal services were born in the UK. Moreover, the likelihood of help-seeking is influenced by the mode of onset of psychotic symptoms
[37]. Previous studies showed that patients in less symptomatic states were more likely to seek help from their GPs [37]. Considering that $44 \%$ of all our FEP patients had an acute onset of psychotic symptoms, it is not surprising that many would not have sought help via GPs and thus would have not accessed the prodromal services.

The age of first contact was younger in FEP patients who had first presented to the prodromal services and subsequently transitioned to psychotic disorder than in the FEP group without prior contact with prodromal services. Even though these results could be an artefact of the age limit imposed by prodromal services, they may well indicate that the prodromal services are successful in reaching out to younger clients. There was some evidence that DUP differed between the groups, but small samples means this did not reach $P<0.05$; therefore caution is needed when drawing conclusions. It has been shown that the mode of onset of first psychotic symptoms is one of the strongest determining factors of length of DUP [31]. Indeed, the vast majority of our prodromal group had an insidious mode of onset of their first psychotic symptoms. With slow onset of first symptoms it may be difficult to distinguish the first indicators of the illness from other motivational or developmental difficulties [32, 43]. Further we note that 'insidious onset' could also encompass onset with predominantly negative symptoms. However, such symptoms were not specifically rated. Members of their families or close friends may be also less likely to encourage these individuals to seek help when the onset of symptoms is spread over a long period [32]. It is possible of course that treatment delay could have been even longer for these individuals had it not been for the presence of prodromal services. 
The evidence from the literature suggests that the type of professional with whom the first contact is made following onset of psychosis is an important factor in determining the length of DUP [20, 41, 43]. Considering that GP attendance is associated with a prolonged DUP [41], while the emergency medical services and criminal justice agency are associated with a substantially shorter DUP [20], it may be that those who have an acute onset are likely to access care quickly and, by virtue of being already psychotic, tend to bypass the prodromal services. Given this, prodromal services appear to be the services for slow developing psychosis.

Nonetheless, a recent retrospective study conducted in South London [21] showed that those FEP patients who presented to the prodromal services up to one year before making the transition to a psychotic disorder had a median DUP of 7 days, substantially shorter than the one we observed (median 19 days). However, these differences can be explained by differences in measurement and problems inherent to the DUP construct. The claim that early intervention services reduce DUP relative to generic clinical services [21] is critically dependent on whether the time between the earliest report of symptoms and the beginning of the first treatment under care of early intervention services is taken as the DUP. Alternatively, the beginning of DUP is taken as a 'reset' after such an intervention unless or until the individual subsequently develops their first episode of clinical psychosis. One approach that would help avoid this problem is to clearly differentiate between the duration of the prodromal period, defined as the period from the first unspecific symptoms related to psychosis to the first continuous (present most of the time) psychotic symptom [44] and the actual DUP. This method would highlight whether and how much the prodromal services benefit the patients before they make the transition to a FEP and how lasting these benefits are over the subsequent course of illness. Unfortunately, we were unable to examine duration of prodromal period in the present study.

Additionally, we found that around $75 \%$ of all patients referred to the prodromal services and who ultimately went psychotic, were already experiencing their first episode psychosis at the time of contact with the prodromal services. Although this supports the notion that the prodromal services are successful in detecting FEP patients who are in turn promptly referred to more appropriate early intervention services, the results of the present study do not suggest that the prodromal services provide additional functions by detecting individuals with incipient first onset of psychosis who otherwise would not have had access to mental health services.

\section{Methodological considerations}

This is the first study to investigate the differences in pathways to care and clinical presentation between heterogeneous groups of FEP patients resident in inner city deprived areas of South East London. We utilized a large sample size of well-characterised incidence cases from both inpatient and outpatient settings, which is representative of actual clinical practice. The SLaM BRC Case Register, which was the primary source of information for the present study, has a near 100\% clinical coverage in its boroughs [24]; this further enhanced the generalizability of our findings.

The results of the present study should be interpreted in light of methodological limitations. It may be argued that extracting information from clinical records may not always produce reliable data. For example, for the purposes of determining DUP from clinical records, treating clinicians might not always have recorded in the notes when psychotic symptoms began and their magnitude, thus potentially introducing bias. Although we utilised an operationalised definition of DUP, we did not undertake reliability checks of the information used in this construct. Having said that the distribution of DUP reported in this study is consistent with other research [45-47]. The quality and completeness of information recorded in the electronic notes for each patient inevitably varied and this may have introduced some bias. Further, case register information is limited to people who sought help for their symptoms and thus have accessed services. This however excludes undiagnosed mentally ill individuals within the community. It is also feasible that some of the patients might have sought or purchased mental healthcare elsewhere for a psychotic disorder and thus would not have been registered in the SLaM BRC Case Register, nor included in the present study. The number of patients with FEP who came through the prodromal services was relatively small; therefore, caution is needed when interpreting the results. Finally, the data relating to living circumstances, relationship status and employment provide crude proxies for social networks and as such they can only hint at the potential role of social contexts and networks in influencing the pathway to care.

\section{Conclusion}

Under the current pathways to care, only a small fraction of individuals (4.1\%) who present with a FEP to the main secondary mental health provider actually come in contact with the prodromal services before making a subsequent transition to a psychotic disorder. Much of the work of the prodromal services, and by implication similar prodromal programmes, is spent dealing with people who either will never become psychotic or alternatively are already in their FEP. While this signifies an appetite for a variety of 
flexible services to care for people with early psychosis, it highlights a greater challenge in providing care for people before they develop psychosis and to therefore prevent it or catch it early. Maintaining contact with all such people and responding when interventions are required takes considerable resources. Our findings also imply that research based on the view that at-risk patients recruited through prodromal services capture a process or phase in the illness that is typical of the majority of FEP patients may be questioned.

\section{Additional files}

Additional file 1: Comparisons in socio-demographic characteristics on presentation at first contact with mental health services between FEP-C (control) and FEP-P (psychosis) groups (DOCX $18 \mathrm{~kb}$ )

Additional file 2: Comparisons in clinical presentation characteristics and pathways to care between FEP-C (control) and FEP-P (psychosis) groups (DOCX $16 \mathrm{~kb}$ )

\section{Abbreviations}

ARMS: At Risk Mental State; BRC: Biomedical Research Centre; CRIS: Case Register Interactive Search tool; DUP: Duration of untreated psychosis: EU-GEl: European Union Gene-Environment Interactions; FEP: First episode of psychosis; GP: General Practitioner; ICD: International Classification of Diseases; IQR: Inter-quartile range; SCAN: Schedules for Clinical Assessment in Neuropsychiatry; SLaM: South London and Maudsley NHS Foundation Trust; WHO: World Health Organisation

\section{Acknowledgements \\ CRIS is supported by the National Institute of Health Research (NIHR) Biomedical Research Centre for Mental Health, BRC Nucleus at the South London and Maudsley NHS Foundation Trust and Institute of Psychiatry, King's College London jointly funded by the Guy's and St Thomas' Trustees and the South London and Maudsley Trustees. We are grateful to our colleagues in the prodromal and early intervention service. The views expressed are those of the author(s) and not necessarily those of the National Health Service (NHS), the NIHR or the Department of Health. \\ Funding \\ The EU-GEl study is funded by the European Community's Seventh Framework Programme under grant agreement (No. HEALTH-F2-2010-241,909). This project was supported by the National Institute of Health Research, Biomedical Research Centre for Mental Health, at South London \& Maudsley NHS Foundation Trust and King's College London.}

\section{Availability of data and materials}

The datasets used and/or analysed during the current study are available from the corresponding author on reasonable request.

\section{Authors' contributions}

OA was responsible for to extracting data from CRIS, running the data analyses, designing figures and writing the manuscript. CG-A, SO, FB, SB, JW contributed to data extraction and literature search. JHM and PD were involved in drafting the manuscript. CM, RMM and ASD. designed and coordinated the study, were involved in drafting and revising the manuscript. All authors approved the final manuscript.

\section{Authors' information}

O.A. is a Postdoctoral Researcher in Psychiatric Research; C.M. is Professor of Social Epidemiology; C.G.-A. is a Postdoctoral Researcher; S.O. is a project manager; F.B. is a PhD candidate; S.B. is a medical student; J.W. is a post-graduate student; J.H.M. is Reader in the Epidemiology of Psychosis; P.D. is Professor of Neurobiology of Psychosis; R.M.M. is Professor of Psychiatric Research; A.S.D. is Professor of Cognitive Neuropsychiatry.

\section{Ethics approval and consent to participate}

The SLaM BRC Case Register Clinical Record Interactive Search system (CRIS) system was approved as an anonymised dataset for secondary analysis by the Oxfordshire Research Ethics Committee (reference 08/H0606/71). Similar to previous studies that utilised the CRIS system [48], and in compliance with European data protection law individual consent was not obtained for this study because data had been effectively anonymised by CRIS prior to access.

\section{Consent for publication}

Not applicable.

\section{Competing interests}

R.M.M. and A.S.D. receive salary support from the NIHR BRC. R.M.M. has received honoraria from Janssen, Astra-Zeneca, Lilly, and BMS. A.S.D. has received honoraria from Janssen Pharmaceuticals.

\section{Publisher's Note}

Springer Nature remains neutral with regard to jurisdictional claims in published maps and institutional affiliations.

\section{Author details}

'Department of Psychosis Studies, Institute of Psychiatry, Psychology \& Neuroscience, King's College London, 16 De Crespigny Park, London SE5 8AF, UK. ${ }^{2}$ Society and Mental Health Research Group, Health Service and Population Research Department, Institute of Psychiatry, Psychology \& Neuroscience, King's College London, De Crespigny Park, London SE5 8AF, UK. ${ }^{3} \mathrm{NIHR}$ Biomedical Research Centre, David Goldberg Building, Institute of Psychiatry, Psychology \& Neuroscience, Kings College London, De Crespigny Park, London SE5 8AF, UK. ${ }^{4}$ Guy's, King's and St Thomas' School of Medical Education, King's College London, London, UK. ${ }^{5}$ Violence Prevention Research Unit Queen Mary University of London \& East London NHS Foundation Trust, Garrod Building, Turner Street, London E1 2AD, UK. ${ }^{6}$ National Institute for Health Research (NIHR) Mental Health Biomedical Research Centre at South London and Maudsley NHS Foundation Trust and King's College London, London, UK.

Received: 14 June 2017 Accepted: 15 August 2017

Published online: 25 August 2017

\section{References}

1. Drake RJ, Haley CJ, Akhtar S, Lewis SW. Causes and consequences of duration of untreated psychosis in schizophrenia. Br J Psychiatry. 2000;177:511-5.

2. Üçok A, Serbest S, Kandemir PE. Remission after first-episode schizophrenia: results of a long-term follow-up. Psychiatry Res. 2011;189(1):33-7.

3. Tang JYM, Chang WC, Hui CLM, Wong GHY, Chan SKW, Lee EHM, Yeung WS, Wong CK, Tang WN, Chan WF, et al. Prospective relationship between duration of untreated psychosis and 13-year clinical outcome: a firstepisode psychosis study. Schizophr Res. 2014;153(1-3):1-8.

4. Bosanac P, Patton GC, Castle DJ. Early intervention in psychotic disorders: faith before facts? Psychol Med. 2010 Mar;40(3):353-8.

5. Agius M, Hadjinicolaou AV, Ramkisson R, Shah S, Haq SU, Tomenson B, Zaman R. Does early intervention for psychosis work? An analysis of outcomes of early intervention in psychosis based on the critical period hypothesis, measured by number of admissions and bed days used over a period of six years, the first three in an early intervention service, the second three in a community mental health team. Psychiatr Danub. 2010;22(1):S72-84.

6. Birchwood M, Todd P, Jackson C. Early intervention in psychosis. The critical period hypothesis. Br J Psychiatry Suppl. 1998;172(33):53-9.

7. Johannessen JO, Larsen TK, Joa I, Melle I, Friis S, Opjordsmoen S, Rund BR, Simonsen E, Vaglum P, McGlashan TH. Pathways to care for first-episode psychosis in an early detection healthcare sector: part of the Scandinavian TIPS study. Br J Psychiatry Suppl. 2005;48(8):s24-8.

8. Singh SP, Grange T. Measuring pathways to care in first-episode psychosis: a systematic review. Schizophr Res. 2006;81(1):75-82.

9. Fusar-Poli P, Byrne M, Badger S, Valmaggia LR, McGuire PK. Outreach and support in south London (OASIS), 2001-2011: ten years of early diagnosis and treatment for young individuals at high clinical risk for psychosis. Eur Psychiatry. 2013;28(5):315-26. 
10. Yung AR, Stanford C, Cosgrave E, Killackey E, Phillips L, Nelson B, McGorry PD. Testing the ultra high risk (prodromal) criteria for the prediction of psychosis in a clinical sample of young people. Schizophr Res. 2006;84(1):57-66.

11. Yung AR, Phillips LJ, Yuen HP, Francey SM, McFarlane CA, Hallgren M, McGorry PD. Psychosis prediction: 12-month follow up of a high-risk ("prodromal") group. Schizophr Res. 2003;60(1):21-32.

12. Fusar-Poli P, Bonoldi I, Yung AR, Borgwardt S, Kempton MJ, Valmaggia L, Barale F, Caverzasi E, McGuire P. Predicting psychosis: meta-analysis of transition outcomes in individuals at high clinical risk. Arch Gen Psychiatry. 2012;69(3):220-9

13. Conrad AM, Lewin TJ, Sly KA, Schall U, Halpin SA, Hunter M, Carr VJ. Utility of risk-status for predicting psychosis and related outcomes: evaluation of a 10-year cohort of presenters to a specialised early psychosis community mental health service. Psychiatry Res. 2017;247:336-44.

14. Malla A, de Bonneville M, Shah J, Jordan G, Pruessner M, Faridi K, Rabinovitch M, lyer SN, Joober R. Outcome in patients converting to psychosis following a treated clinical high risk state. Early Interv Psychiatry. 2017;14(10):12431.

15. Kirkbride JB, Fearon P, Morgan C, Dazzan P, Morgan K, Tarrant J, Lloyd T, Holloway J, Hutchinson G, Leff JP, et al. Heterogeneity in incidence rates of schizophrenia and other psychotic syndromes: findings from the 3-center AeSOP study. Arch Gen Psychiatry. 2006;63(3):250-8.

16. Yung AR, McGorry PD, McFarlane CA, Jackson HJ, Patton GC, Rakkar A. Monitoring and care of young people at incipient risk of psychosis. Schizophr Bull. 1996;22(2):283-303.

17. Power P, McGuire P, lacoponi E, Garety P, Morris E, Valmaggia L, Grafton D, Craig T. Lambeth early onset (LEO) and Outreach \& Support in South London (OASIS) service. Early Interv Psychiatry. 2007;1(1):97-103.

18. Green CE, McGuire PK, Ashworth M, Valmaggia LR. Outreach and support in South London (OASIS). Outcomes of non-attenders to a service for people at high risk of psychosis: the case for a more assertive approach to assessment. Psychol Med. 2011:41(2):243-50.

19. von Reventlow HG, Kruger-Ozgurdal S, Ruhrmann S, Schultze-Lutter F, Heinz A, Patterson $P$, Heinimaa M, Dingemans $P$, French $P$, Birchwood $M$, et al. Pathways to care in subjects at high risk for psychotic disorders - a European perspective. Schizophr Res. 2014;152(2-3):400-7.

20. Ghali S, Fisher HL, Joyce J, Major B, Hobbs L, Soni S, Chisholm B, Rahaman $\mathrm{N}$, Papada $\mathrm{P}$, Lawrence J, et al. Ethnic variations in pathways into early intervention services for psychosis. Br J Psychiatry. 2013;202(4):277-83.

21. Fisher HL, Roberts A, Day F, Reynolds N, lacoponi E, Garety PA, Craig TK, McGuire $P$, Valmaggia L, Power P. Impact of crime victimization on initial presentation to an early intervention for psychosis service and 18-month outcomes. Early Interv Psychiatry. 2015;

22. WHO: Schedules for Clinical Assessment in Neuropsychiatry. Version 2 Manual: World Health Organization, Division of Mental Health; 1994.

23. ONS. 2011 Census - Population and Household Estimates for England and Wales, 2011, 2912. In.: Office of National Statistics ONS; 2011.

24. Stewart R, Soremekun M, Perera G, Broadbent M, Callard F, Denis M, Hotopf M, Thornicroft G, Lovestone S. The South London and Maudsley NHS Foundation Trust biomedical research Centre (SLAM BRC) case register: development and descriptive data. BMC Psychiatry. 2009:9(51):9-51.

25. Fernandes AC, Cloete D, Broadbent MT, Hayes RD, Chang CK, Jackson RG, Roberts A, Tsang J, Soncul M, Liebscher J, et al. Development and evaluation of a de-identification procedure for a case register sourced from mental health electronic records. BMC Med Inform Decis Mak. 2013;13(71):1472-6947.

26. Perera G, Broadbent M, Callard F, Chang CK, Downs J, Dutta R, Fernandes A, Hayes RD, Henderson M, Jackson R, et al. Cohort profile of the South London and Maudsley NHS Foundation Trust biomedical research Centre (SLaM BRC) case register: current status and recent enhancement of an electronic mental health record-derived data resource. BMJ Open. 2016;6(3): 2015-008721

27. Yung AR, Yuen HP, McGorry PD, Phillips $\sqcup$, Kelly D, Dell'Olio M, Francey SM, Cosgrave EM, Killackey E, Stanford C, et al. Mapping the onset of psychosis: the comprehensive assessment of at-risk mental states. Aust N Z J Psychiatry. 2005;39(11-12):964-71.

28. Mallett R, Leff J, Bhugra D, Pang D, Zhao JH. Social environment, ethnicity and schizophrenia. A case-control study. Soc Psychiatry Psychiatr Epidemiol. 2002;37(7):329-35.

29. Norman RM, Malla AK. Duration of untreated psychosis: a critical examination of the concept and its importance. Psychol Med. 2001; 31(3):381-400.
30. McKenzie K, Samele C, Van Horn E, Tattan T, Van Os J, Murray R. Comparison of the outcome and treatment of psychosis in people of Caribbean origin living in the UK and British whites. Report from the UK700 trial. Br J Psychiatry. 2001;178:160-5.

31. Compton MT, Chien VH, Leiner AS, Goulding SM, Weiss PS. Mode of onset of psychosis and family involvement in help-seeking as determinants of duration of untreated psychosis. Soc Psychiatry Psychiatr Epidemiol. 2008; 43(12):975-82.

32. Morgan C, Abdul-Al R, Lappin JM, Jones P, Fearon P, Leese M, Croudace T, Morgan K, Dazzan P, Craig T, et al. Clinical and social determinants of duration of untreated psychosis in the AESOP first-episode psychosis study. Br J Psychiatry. 2006;189:446-52.

33. Cuchel M, Bloedon LT, Szapary PO, Kolansky DM, Wolfe ML, Sarkis A, Millar JS, Ikewaki K, Siegelman ES, Gregg RE, et al. Inhibition of microsomal triglyceride transfer protein in familial hypercholesterolemia. N Engl J Med. 2007;356(2):148-56.

34. Heinze G. A comparative investigation of methods for logistic regression with separated or nearly separated data. Stat Med. 2006;25(24):4216-26.

35. Harrell FE Jr, Lee KL, Matchar DB, Reichert TA. Regression models for prognostic prediction: advantages, problems, and suggested solutions. Cancer Treat Rep. 1985;69(10):1071-7.

36. Peduzzi $P$, Concato J, Kemper $E$, Holford TR, Feinstein AR. A simulation study of the number of events per variable in logistic regression analysis. J Clin Epidemiol. 1996:49(12):1373-9.

37. Platz C, Umbricht DS, Cattapan-Ludewig K, Dvorsky D, Arbach D, Brenner HD, Simon AE. Help-seeking pathways in early psychosis. Soc Psychiatry Psychiatr Epidemiol. 2006;41(12):967-74.

38. Shrivastava A. Should "risk syndrome for psychosis" be included as a diagnosis in DSM-V? World Psychiatry. 2010;9(2):123.

39. Lieberman JA, Perkins D, Belger A, Chakos M, Jarskog F, Boteva K, Gilmore J. The early stages of schizophrenia: speculations on pathogenesis, pathophysiology, and therapeutic approaches. Biol Psychiatry. 2001;50(11):884-97.

40. Morgan C, Mallett R, Hutchinson G, Bagalkote H, Morgan K, Fearon P, Dazzan P, Boydell J, McKenzie K, Harrison G, et al. Pathways to care and ethnicity. 2: source of referral and help-seeking. Report from the AESOP study. Br J Psychiatry. 2005;186:290-6.

41. Bechard-Evans L, Schmitz N, Abadi S, Joober R, King S, Malla A. Determinants of help-seeking and system related components of delay in the treatment of first-episode psychosis. Schizophr Res. 2007;96(1-3):206-14.

42. Alvidrez J, Snowden LR, Kaiser DM. The experience of stigma among black mental health consumers. J Health Care Poor Underserved. 2008;19(3):874-93.

43. Tanskanen S, Morant N, Hinton M, Lloyd-Evans B, Crosby M, Killaspy H, Raine R, Pilling S, Johnson S. Service user and carer experiences of seeking help for a first episode of psychosis: a UK qualitative study. BMC Psychiatry. 2011;11(157):11-157.

44. Crespo-Facorro B, Roiz-Santianez R, Pelayo-Teran JM, Gonzalez-Blanch C, Perez-Iglesias R, Gutierrez A, de Lucas EM, Tordesillas D, Vazquez-Barquero $J$. Caudate nucleus volume and its clinical and cognitive correlations in first episode schizophrenia. Schizophr Res. 2007;91(1-3):87-96.

45. Opjordsmoen S, Friis S, Melle I, Haahr U, Johannessen JO, Larsen TK, Rossberg JI, Rund BR, Simonsen E, Vaglum P, et al. A 2-year follow-up of involuntary admission's influence upon adherence and outcome in firstepisode psychosis. Acta Psychiatr Scand. 2010;121(5):371-6.

46. Emsley R, Rabinowitz J, Medori R. Remission in early psychosis: rates, predictors, and clinical and functional outcome correlates. Schizophr Res. 2007;89(1-3):129-39.

47. Perkins DO, Lieberman JA, Gu H, Tohen M, McEvoy J, Green Al, Zipursky RB, Strakowski SM, Sharma T, Kahn RS, et al. Predictors of antipsychotic treatment response in patients with first-episode schizophrenia, schizoaffective and schizophreniform disorders. Br J Psychiatry. 2004;18:18-24.

48. Wu CY, Chang CK, Robson D, Jackson R, Chen SJ, Hayes RD, Stewart R. Evaluation of smoking status identification using electronic health records and open-text information in a large mental health case register. PLoS One. 2013:8(9). 